\title{
Stability and Behaviour of Rotationally Symmetric Harmonic Maps From A Ball Into A Sphere
}

\author{
Chriestie E. J. C. Montolalu ${ }^{1}$ \\ ${ }^{1}$ Program Studi Matematika, FMIPA, UNSRAT Manado, chriestelly@ yahoo.com
}

\begin{abstract}
Rotationally Symmetric Harmonic Maps from a Ball into a Sphere has been studied before. The systems conducted in this study can be analyzed further by checking its stability and its behavior in the system. This paper will show how to determine the stability of the system and its behaviour by reducing it into a damped pendulum equation differential equation.
\end{abstract}

Keywords: Rotational symmetry, Harmonic maps, Stability, Damped pendulum equation.

\section{Kestabilan dan Sifat dari Pemetaan Harmonik yang Berotasi Simetris dari Sebuah Bola ke Sebuah Sphere}

\begin{abstract}
Abstrak
Pemetaan Harmonik yang Berotasi Simmetris dari sebuah bola ke sebuah "sphere" telah dipelajari sebelumnya. Sistem yang ditemukan dalam studi ini bisa dianalisa lebih lanjut dengan memeriksa kestabilan dan sifat-sifatnya dalam sistem. Tulisan ini akan menunjukkan cara untuk menentukan kestabilan sistem dan sifat-sifatnya dengan mengubahnya ke dalam bentuk persamaan differensial pendulum sederhana.
\end{abstract}

Kata kunci:Rotasi simetris, Pemetaan harmonic, Kestabilan, Persamaan pendulum sederhana.

\section{Introduction}

An object with rotational symmetry is an object that looks the same after a certain amount of rotation. A map between two compact Riemannian manifolds is a harmonic map if it is a critical point for the energy functional. For example, a map from a circle to the equator of standard 2sphere is a harmonic map, and so are the maps that take the circle and map it around the equator $n$ times, for any integer $n$.

Let $M$ be a $n$-dimensional Riemannian manifold (with or without boundary) with a smooth Riemannian metric $g$. In a local coordinates around fixed point $p \in M, g$ can be represented by

$$
g=g_{i j} d x_{i} \otimes d x_{j},
$$

where $g_{i j}$ is a positive definite symmetric $n \times n$ matrix. Let $\left(g^{i j}\right)=\left(g_{i j}\right)^{-1}$ be the inverse matrix of $\left(g_{i j}\right)$ and the volume element of $(M ; g)$ is

$$
d v_{g}=\sqrt{|g|} d x
$$

where $|g|=\operatorname{det}\left(g_{i j}\right)$. Let $N$ be another $l$-dimensional compact Riemmanian manifold (without boundary) with a smooth Riemmanian metric $h$.

For a map $u: M \rightarrow N$, its Dirichlet energy functional is defined by

where the density function $e(u)$ is given by

$$
E(u)=\int_{M} e(u) d v_{g}
$$

$$
e(u)(x)=\frac{1}{2}|\nabla u(x)|^{2}=\frac{1}{2} \sum_{\alpha, \beta, i, j} g^{i j}(x) h_{\alpha \beta}(u(x)) \frac{\partial u^{\alpha}}{\partial x_{i}} \frac{\partial u^{\beta}}{\partial x_{j}}
$$

A smooth map $u$ from $M$ to $N$ is said to be a harmonic map if $u$ is a critical point of the Dirichlet energy functional $E$; i.e. it satisfies 


$$
\Delta_{M} u+A(u)(\nabla u, \nabla u)=0
$$

in $M$, where $\Delta_{M}$ is the Laplacian operator with respect to the Riemmanian metric of $M$ and $A$ is the second fundamental form of $M$.

In this case, the harmonic map was considered to be from unit ball to unit sphere which satisfies a variational problem in Euler equation form:

$$
-\Delta u=|\nabla u|^{2} . u \text {. }
$$

Let $\kappa \geq 0$ be an upper bound for the sectional curvature of $N$ and $K_{p}(q)$ the open geodesic ball in $N$ with center $q$ and radius $\rho$. Assuming essentially the size restriction

$$
f(\partial M) \subset K_{p}(q), \rho \leq \frac{\pi}{2 \sqrt{\kappa}},
$$

Hilderbrandt et. Al. [5] showed existence of a "small" smooth harmonic maps satisfying (1.2). This was shown by considering solution of a Dirichlet problem, which is smooth in the interior and minimizes the energy in the class in $K_{p}(q)$ having the boundary values $f$. In the case where $N$ is the standard sphere the smallness condition restricts the image of the boundary values and the solution to an open half-sphere.

Suppose $B^{n}$ denotes the compact unit ball in the Euclidean space $R^{n}$, and $S^{n}$ the unit sphere in $R^{n+1}$. Select the point given by the $(n+1)$-th standard base vector $e_{n+1}$ in $R^{n+1}$ as northpole of the sphere. Every map $u: B^{n} \rightarrow S^{n}$ can be written in the form

with maps

$$
u(x)=(g(x) \cdot \sin \varphi(x), \cos \varphi(x))
$$

$$
\varphi: B^{n} \rightarrow[0, \pi], \quad g: B^{n} \rightarrow S^{n-1} \subset R^{n}
$$

$\varphi$ measures the Riemannian distance from $u(x)$ to the northpole on the sphere and is called radius function of $u$. The map $g$ is uniquely defined by $u$ except for points $x$ where $u(x)= \pm e_{n+1}$. A map $u: B^{n} \rightarrow S^{n}$ is rotationally symmetric if and only if

$$
g(x)=\frac{x}{|x|} \text { and } \varphi(x)=\Phi(|x|) .
$$

In order to study the question what happens if the smallness condition is violated, the family of boundary values can be defined as

$$
f_{\rho}: \partial B^{n} \rightarrow S^{n}, f_{\rho}(x)=(x \cdot \sin \rho, \cos \rho)
$$

which is considered as an homotopy with parameter $\rho \in[0, \pi]$. For $n=2$ as shown by Lemaire in [3] and $n \geq 3$ by Wood in [1], harmonic maps from $B^{n}$ into a Riemannian manifold are constant if they are constant on the boundary. Therefore, for $\rho=0$ and $\rho=\pi$ the Dirichlet problem has exactly one trivial solution.

In this project, the harmonic maps were restricted to rotationally symmetric behavior. The stability of the result will be shown by reducing the discussion to ordinary differential equation, especially to the damped pendulum equation.

\section{Rotationally Symmetric Harmonic Maps}

We are going to study rotationally symmetric maps with finite energy. Recall that every map $u: B^{n} \rightarrow S^{n}$ can be written in the form

with maps

$$
u(x)=(g(x) \cdot \sin \varphi(x), \cos \varphi(x))
$$

$$
\varphi: B^{n} \rightarrow[0, \pi], g: B^{n} \rightarrow S^{n-1} \subset R^{n}
$$

$\varphi$ measures the Riemannian distance from $u(x)$ to the northpole on the sphere and is called radius function of $u$. A map $u: B^{n} \rightarrow S^{n}$ is rotationally symmetric if and only if

$$
g(x)=\frac{x}{|x|} \text { and } \varphi(x)=\Phi(|x|) .
$$

In this case, metric form of the manifold can be written as [2]:

$$
|u|^{2}=|\varphi|^{2}+f^{2}(\varphi) \cdot|g|^{2}
$$

Since the projection assumed is projection from unit ball to unit sphere, then this can be assumed as a function in geodesic coordinate of a sphere, i.e. $f(\varphi)=\sin \varphi$.

Therefore, for $u(x)=\left(\frac{x}{r} \sin \Phi(r), \cos \Phi(r)\right)$, with radius function $\Phi:[0,1] \rightarrow[0, \pi]$ depending only on $r=|x|$, the metric form can be written as 
And

$$
|u|^{2}=|\Phi(r)|^{2}+\sin ^{2}(\Phi(r)) \cdot\left|\frac{x}{r}\right|^{2}
$$

Suppose $\Psi(t)=\Phi\left(e^{t}\right), \Psi:(-\infty, 0] \rightarrow[0, \pi]$

$$
|\nabla u|^{2}=|\nabla \Phi(r)|^{2}+\sin ^{2}(\Phi(r)) \cdot\left|\nabla\left(\frac{x}{r}\right)\right|^{2}
$$

$\Psi^{\prime}(t)=e^{t} \Phi^{\prime}\left(e^{t}\right)$

$\Psi^{\prime \prime}(t)=e^{t} \Phi^{\prime}\left(e^{t}\right)+e^{2 t} \Phi^{\prime \prime}\left(e^{t}\right)$

and can be written in differential equation $[2,4]$ as follows:

$\Psi^{\prime \prime}(t)+(n-2) \Psi^{\prime}(t)-\frac{n-1}{2} \sin 2 \Psi(t)=0$

The energy can be expressed in term of $\Psi(t)$ :

$$
\begin{aligned}
E(u) & =\frac{\omega_{n}}{2} \int_{-\infty}^{0}\left[\Phi^{\prime 2}\left(e^{t}\right)+\frac{n-1}{e^{2 t}} \sin ^{2} \Phi\left(e^{t}\right)\right] e^{(n-1) t} e^{t} d t \\
& =\frac{\omega_{n}}{2} \int_{-\infty}^{0}\left[e^{2 t} \Phi^{\prime 2}\left(e^{t}\right)+(n-1) \sin ^{2} \Phi\left(e^{t}\right)\right] e^{-2 t} e^{(n-1) t} e^{t} d t \\
& =\frac{\omega_{n}}{2} \int_{-\infty}^{0}\left[\Psi^{\prime 2}(t)+(n-1) \sin ^{2} \Psi(t)\right] e^{(n-2) t} d t
\end{aligned}
$$

The differential equation (2.3) can be written in damped pendulum equation form as follows.

Set $q(t)=2 \Psi(t)-\pi$ and $p(t)=q^{\prime}(t)$, then

$$
\left(\begin{array}{c}
q^{\prime} \\
p^{\prime}
\end{array}\right)=\left(\begin{array}{c}
p \\
-(n-2) p-(n-1) \sin q
\end{array}\right)
$$

The critical points of this system, i.e. when $p^{\prime}(t)=q^{\prime}(t)=0$, imply critical points in phase plane $\left(\Psi(t), \Psi^{\prime}(t)\right)$, which are $(0,0),(\pi, 0)$, and $\left(\frac{\pi}{2}, 0\right)$.

Here, we have to study those solutions corresponding to harmonic maps with finite energy. In this analogy the equator corresponds to the stable respoint, whereas the northpole and the southpole correspond to unstable restpoint.

Consider a function: $V(t)=\left(\Psi^{\prime}(t)\right)^{2}-(n-1) \sin ^{2} \Psi(t)$

Then

$$
\begin{aligned}
V^{\prime}(t) & =2 \Psi^{\prime}(t) \cdot \Psi^{\prime \prime}(t)-2(n-1) \Psi^{\prime}(t) \cdot \sin \Psi(t) \cdot \cos \Psi(t) \\
& =2 \Psi^{\prime}(t) \cdot\left[\Psi^{\prime \prime}(t)-\frac{(n-1)}{2} \sin 2 \Psi(t)\right]
\end{aligned}
$$

By (2.3), $\Psi^{\prime \prime}(t)-\frac{n-1}{2} \sin 2 \Psi(t)=-(n-2) \Psi^{\prime}(t)$, thus

$$
\begin{aligned}
V^{\prime}(t) & =2 \Psi^{\prime}(t) \cdot\left[-(n-2) \Psi^{\prime}(t)\right] \\
& =-2(n-2)\left(\Psi^{\prime}(t)\right)^{2}
\end{aligned}
$$

for $n \geq 2, V^{\prime}(t) \leq 0$

Since $\Psi:(-\infty, 0] \rightarrow[0, \pi]$, then $\sin ^{2} \Psi(t) \rightarrow 0$

This means $V(t) \geq 0$

These properties, i.e. $V(t) \geq 0$ and $V^{\prime}(t) \leq 0$, satisfy the properties of a Lyapunov function. Therefore, $V(t)$ is a Lyapunov function for the differential equation.

Equivalently, for $V(t) e^{(n-2) t},\left(V(t) e^{(n-2) t}\right)^{\prime}$ can be written as

$$
\left(V(t) e^{(n-2) t}\right)^{\prime}=-(n-2)\left[\Psi^{\prime 2}(t)+(n-1) \sin ^{2} \Psi(t)\right] e^{(n-2) t}
$$

For $E(u)<\infty$,

Then because of (2.8)

$$
\liminf _{t \rightarrow-\infty}\left[\Psi^{\prime 2}(t)+(n-1) \sin ^{2} \Psi(t)\right] e^{(n-2) t}=0
$$

$$
\lim _{t \rightarrow-\infty} V(t) e^{(n-2) t}=0
$$

and by partial integration, $E(u)$ can be written as: 


$$
\begin{aligned}
E(u) & =\frac{\omega_{n}}{2} \int_{-\infty}^{0} \frac{\left(V(t) e^{(n-2) t}\right)^{\prime}}{-(n-2)} d t \\
& =-\frac{\omega_{n}}{2(n-2)} \int_{-\infty}^{0} V^{\prime}(t) e^{(n-2) t}+(n-2) V(t) e^{(n-2) t} d t \\
& =-\frac{\omega_{n}}{2(n-2)}\left[\int_{-\infty}^{0} V^{\prime}(t) e^{(n-2) t} d t+\int_{-\infty}^{0}(n-2) V(t) e^{(n-2) t} d t\right]
\end{aligned}
$$

It is clear that

$$
\int_{-\infty}^{0} V^{\prime}(t) e^{(n-2) t} d t=\left.V(t) e^{(n-2) t}\right|_{-\infty} ^{0}-\int_{-\infty}^{0}(n-2) V(t) e^{(n-2) t} d t
$$

Thus,

$$
E(u)=-\frac{\omega_{n}}{2(n-2)}\left[\left.V(t) e^{(n-2) t}\right|_{-\infty} ^{0}\right]
$$

Since $\lim _{t \rightarrow-\infty} V(t) e^{(n-2) t}=0$

$$
\begin{aligned}
E(u) & =-\frac{\omega_{n}}{2(n-2)}[V(0)] \\
& =-\frac{\omega_{n}}{2(n-2)}\left[\left(\left(\Psi^{\prime}(0)\right)^{2}-(n-1) \sin ^{2} \Psi(0)\right)\right] \\
& =\frac{\omega_{n}(n-1)}{2(n-2)}\left[\sin ^{2} \Psi(0)-\frac{{\psi^{\prime}}^{2}(0)}{n-1}\right]
\end{aligned}
$$

These results can be used to classify the rotationally symmetric maps of finite energy as follows.

\section{Lemma 1}

Let $\Phi(r)=\Psi(\ln r)$ be the radius function of a rotationally symmetric harmonic map $u$ with finite energy. Then the following cases are possible:

For $n=2$

(i) $2 \arctan (c . r)=\Phi(r)$ or $\pi-\Phi(r)$ with some constant $c \geq 0$

For $n \geq 3$, either

(ii) $\Psi$ is constant with values $0, \frac{\pi}{2}$ or $\pi$,

or

(iii) $\Psi$ is extendable to a solution of the differential equation

$$
\Psi^{\prime \prime}(t)+(n-2) \Psi^{\prime}(t)-\frac{n-1}{2} \sin 2 \Psi(t)=0
$$

and

$\lim _{t \rightarrow-\infty}\left(\Psi(t), \Psi^{\prime}(t)\right)=(0,0)$ or $(\pi, 0)$

$\lim _{t \rightarrow \infty}\left(\Psi(t), \Psi^{\prime}(t)\right)=\left(\frac{\pi}{2}, 0\right)$

\section{Proof}

We extend $\Psi$ to a solution on $R$, which is possible due to the linear growth of the right hand side of (2.5).

\#For $n=2$

$n=2$ in (2.9) gives $\lim _{t \rightarrow-\infty} V(t) e^{0}=0$ which implies $\lim _{t \rightarrow-\infty} V(t)=0$,

and therefore the equation in (3.8) becomes

$$
\begin{aligned}
& \left(\Psi^{\prime}(t)\right)^{2}-\sin ^{2} \Psi(t)=0 \\
& \Leftrightarrow \Psi^{\prime}(t)= \pm \sin \Psi(t) \\
& \Psi^{\prime}(t)=+\sin \Psi(t) \\
& \frac{d \Psi}{d t}=\sin \Psi(t)
\end{aligned}
$$

By using integration by part, this can be written as 
$\int \frac{1}{\sin \Psi} d \Psi=\int d t$

Since $\int \frac{1}{\sin \Psi} d \Psi=\ln \left|\tan \frac{\Psi}{2}\right|+k$

which means

$\ln \left|\tan \frac{\Psi}{2}\right|=t+k$, for some constant $k$

$\tan \frac{\Psi}{2}=e^{t} . c, c=e^{k}$

* Thus, $\Psi(t)=2 \arctan \left(e^{t} . c\right)$

$\Psi^{\prime}(t)=-\sin \Psi(t)=\sin (\pi-\Psi(t))$

$\frac{d \Psi}{d t}=\sin (\pi-\Psi)$

By using integration by part, this can be written as

$\int \frac{1}{\sin (\pi-\Psi)} d \Psi=\int d t$

Since $\int \frac{1}{\sin (\pi-\Psi)} d \Psi=\ln \left|\tan \frac{\pi-\Psi}{2}\right|+k$

which means

$\ln \left|\tan \frac{\pi-\psi}{2}\right|=t+k$, for some constant $k$

$\tan \frac{\pi-\psi}{2}=e^{t} . c, c=e^{k}$

** Thus, $\pi-\Psi(t)=2 \arctan \left(e^{t} . c\right)$

$\therefore *$ and $* *$ are equivalent to case (i).

\# For $n \geq 3$,

$n \geq 3$ in (2.9), which implies $\lim _{t \rightarrow-\infty} V(t)=0$, and in (2.7), which implies $V^{\prime}(t)<0$, give: $\Psi^{\prime 2}(t)<(n-1) \cdot \sin ^{2} \Psi(t)$

This ensures that the phase curve in $\left(\Psi(t), \Psi^{\prime}(t)\right)$ is bounded.

By the definition of Lyapunov function and limit cycles, if $V^{\prime}(t)<0$, then the critical points of the differential system are stable. This means all the trajectories in phase portrait $\left(\Psi(t), \Psi^{\prime}(t)\right)$ converge to the critical points. This is equivalent to case (ii).

Excluding case (ii) gives

$$
\lim _{t \rightarrow-\infty} \Psi(t)=0 \text { or } \pi \text { and } \lim _{t \rightarrow \infty} \Psi(t)=\frac{\pi}{2}
$$

which are equivalent to case (iii).

It is clear that $\Phi(r)=\Psi(\ln r)$ satisfying one of the cases (i) - (iii) lead to harmonic maps with finite energy.

\section{Stability}

The result for $n=2$ in lemma 1 explicitly shows all smooth rotationally symmetric harmonic maps with finite energy.

Whereas for $n \geq 3$, the result in lemma 1 shows that they are directly related to the trajectories of the equation connecting the critical points $(0,0)$ and $(\pi, 0)$ with $\left(\frac{\pi}{2}, 0\right)$ in the phase plane $\left(\Psi(t), \Psi^{\prime}(t)\right)$.

Now consider the critical points in $(q, p)$-plane. They are $(-\pi, 0),(0,0)$ and $(\pi, 0)$. For $n \geq 3$, the critical points can be restricted to $(-\pi, 0)$ and $(0,0)$ corresponding to the northpole and the equator and their connecting trajectories.

The behavior of the system (2.5) in the neighborhood of the critical points is determined by the linearized system as follows. 


\section{Linearize the system around critical point}

A Jacobian matrix: $\left(\begin{array}{ll}\frac{\partial f}{\partial x_{1}} & \frac{\partial f}{\partial x_{2}} \\ \frac{\partial g}{\partial x_{1}} & \frac{\partial g}{\partial x_{2}}\end{array}\right)$

Then, $J=\left(\begin{array}{ll}\frac{\partial f}{\partial q} & \frac{\partial f}{\partial p} \\ \frac{\partial g}{\partial q} & \frac{\partial g}{\partial p}\end{array}\right)=\left(\begin{array}{cc}0 & 1 \\ -(n-1) \cos q & -(n-2)\end{array}\right)$

For $(0,0)$

$J=\left(\begin{array}{cc}0 & 1 \\ -(n-1) & -(n-2)\end{array}\right)$

with eigenvalues

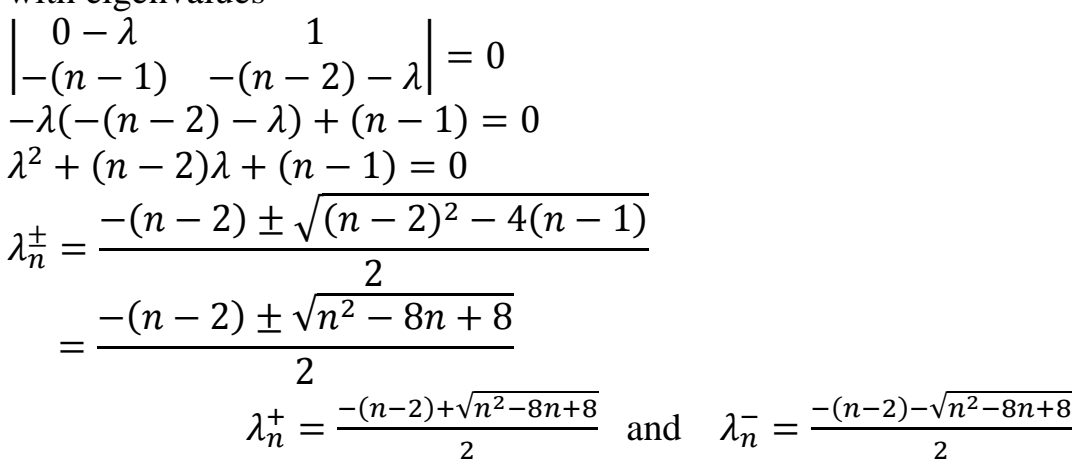

And for $(-\pi, 0)$

$J=\left(\begin{array}{cc}0 & 1 \\ (n-1) & -(n-2)\end{array}\right)$

With eigenvalues

$\left|\begin{array}{cc}0-\Lambda & 1 \\ (n-1) & -(n-2)-\Lambda\end{array}\right|=0$

$-\Lambda(-(n-2)-\Lambda)-(n-1)=0$

$\Lambda^{2}+(n-2) \Lambda-(n-1)=0$

$\Lambda_{n}^{ \pm}=\frac{-(n-2) \pm \sqrt{(n-2)^{2}+4(n-1)}}{2}$

$=\frac{-(n-2) \pm{\sqrt{n^{2}}}^{2}}{2}$

$\Lambda_{n}^{+}=\frac{-(n-2)+n}{2}=\frac{2}{2}=1$ and $\Lambda_{n}^{-}=\frac{-(n-2)-n}{2}=\frac{-2 n+2}{2}=-n+1$

Recall that the behavior and stability of the critical points in phase plane satisfy following properties [4]:

(i) If the eigenvalues of the linearized matrix are real and distinct, then the solution of the system will be:

- stable whenever both of the eigenvalues are both negative. The critical point is a stable improper node.

- unstable whenever one or both of the eigenvalues are positive. The critical point is a saddle.

(ii) If the eigenvalues of the linearized matrix are complex, then the solution for the system will be:

- asymptotically stable if the real part is negative. The critical point is a stable spiral or focus.

- stable if the real part equals to zero. The critical point is a center.

- unstable if the real part is positive. The critical point is a unstable spiral or focus.

(iii) If the eigenvalues of the linearized matrix are real and equal, then the solution for the system will be:

- asymptotically stable if the eigenvalue is negative.

- unstable the eigenvalue greater than and equals to zero. 
The critical point is a proper node if the eigenvalues have two linearly independent eigenvectors, and a degenerate or inflected node if the eigenvalues have one corresponding eigenvector.

Therefore, the stability and behavior of the critical points in this case can be determined as follows: For $(0,0)$ with eigenvalues: $\lambda \frac{ \pm}{n}=\frac{-(n-2) \pm \sqrt{n^{2}-8 n+8}}{2}$,

- if $n=2, \lambda_{n}^{ \pm}= \pm 2 i$, which are purely imaginary, then $(0,0)$ is a center, which means the trajectories in phase plane go around $(0,0)$.

- if $3 \leq n \leq 6, \lambda \frac{ \pm}{n}$ are complex with negative real part, then $(0,0)$ is a stable spiral or focus, which means the trajectories are spiraling into $(0,0)$.

- if $n \geq 7, \lambda_{n}^{ \pm}$are distinct real and negative, then $(0,0)$ is an stable improper node, which means the trajectories are sinking into $(0,0)$. The directions of the trajectories depend on the eigenvector, i.e. they sink into $(0,0)$ from the directions of eigenvector that corresponds to the smallest eigenvector.

For $(-\pi, 0)$ with eigenvalues: $\Lambda_{n}^{+}=1$ and $\Lambda_{n}^{-}=-n+1$, since $n \geq 2, \Lambda_{n}^{-}$will always be negative, then $(-\pi, 0)$ will always be a saddle.

It is clear that there exists an invariant curve in the trajectories between the critical points. For example for $n=3$, the $q p$-plane can be seen in picture 1 . As it is shown in the picture, there exists exactly one invariant curve from $(-\pi, 0)$ to $(0,0)$. The other curves of trajectories can be assumed as a translation in the parameter $t$. Let $(Q, P): R \rightarrow R^{2}$ be such a trajectory, then

$$
\lim _{t \rightarrow-\infty} e^{-t} \cdot P(t)=c
$$

exists and the curve of each trajectory lines up independently, thus $c$ determines $(Q, P)$ uniquely.

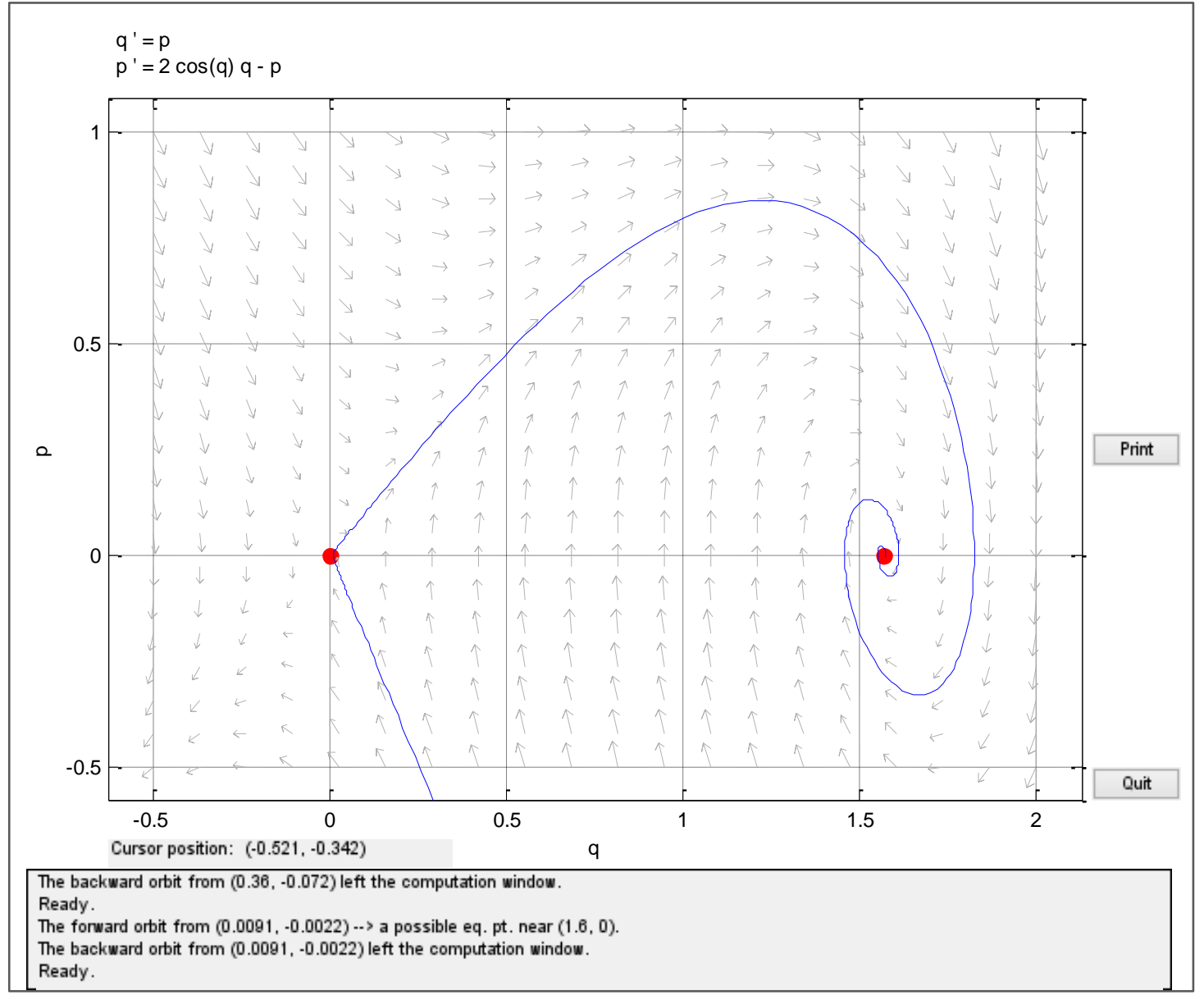

Picture 1. Trajectories in $q p$-plane 


\section{Conclusion}

Study of rotationally symmetric maps with finite energy by considering map $u: B^{n} \rightarrow S^{n}$ that can be written in the form

$$
u(x)=(g(x) \cdot \sin \varphi(x), \cos \varphi(x))
$$

can be reduced into a damped pendulum equation system as follows.

$$
\left(\begin{array}{c}
q^{\prime} \\
p^{\prime}
\end{array}\right)=\left(\begin{array}{c}
p \\
-(n-2) p-(n-1) \sin q
\end{array}\right)
$$

The solution of this system for $n=2$ explicitly shows all smooth rotationally symmetric harmonic maps with finite energy. Whereas for $n \geq 3$, the result shows that they are directly related to the trajectories of the equation connecting the critical points $(0,0)$ and $(\pi, 0)$ with $\left(\frac{\pi}{2}, 0\right)$ in the phase plane $\left(\Psi(t), \Psi^{\prime}(t)\right)$.

Consider the critical points in $(q, p)$-plane: $(-\pi, 0),(0,0)$ and $(\pi, 0)$. For $n \geq 3$, the critical points can be restricted to $(-\pi, 0)$ and $(0,0)$ corresponding to the northpole and the equator and their connecting trajectories.

Therefore, the stability and behavior of the critical points in this case can be determined as follows: For $(0,0)$,

- if $n=2,(0,0)$ is a center, which means the trajectories in phase plane go around $(0,0)$.

- if $3 \leq n \leq 6,(0,0)$ is a stable spiral or focus, which means the trajectories are spiraling into $(0,0)$.

- if $n \geq 7,(0,0)$ is an stable improper node, which means the trajectories are sinking into $(0,0)$. The directions of the trajectories depend on the eigenvector, i.e. they sink into $(0,0)$ from the directions of eigenvector that corresponds to the smallest eigenvector.

For $(-\pi, 0)$, it will always be a saddle.

\section{References}

[1] Wood, J.C, 1981. Non-existence of solutions to certain Dirichlet problems for harmonic maps. I, Preprint.

[2] Evans, L.C. 2010. Partial Differential Equations, American Mathematical Society.

[3] Lemaire, L. 1978. Applications Harmoniques de Surfaces Riemannienes, J. Differential Geom. 13(1978):51-78.

[4] Hartman, P. 1964. Ordinary Differential Equations, New York.

[5] Hilderbrandt, S., H. Kaul, and K. O. Widman. 1977. An Existence Theorem for Harmonic Mappings of Riemmanian Manifolds, Acta. Math. 138(1977): 1-16. 